\title{
Recreating the blood-CNS barrier in vitro
}

Stem-cell-derived organoids reproduce the in vivo secretory and functional properties of the choroid plexus.

A major component of the central nervous system (CNS) is the choroid plexus (ChP), which plays an important role in brain development and function by producing cerebrospinal fluid (CSF). For the first time, researchers at the MRC Laboratory of Molecular Biology have established ChP organoids in vitro to shed light on this rarely studied structure of the brain.

"Like many new directions, the project was the result of serendipity in the beginning. We noticed the presence of choroid plexus regions in our cerebral organoids, and after further investigations became interested in generating more pure choroid plexus organoids to understand CSF production and the important role of the choroid plexus in the brain barrier," said Madeline Lancaster, lead researcher of the study.

The researchers first optimized a protocol to differentiate pluripotent stem cells into ChP organoids and demonstrated that these cells show a striking similarity to human embryonic ChP samples. Compared to a control, $\mathrm{ChP}$ organoids were not only enriched in cuboidal epithelium similar to that of the human ChP but could also recapitulate the transcriptomic signatures of the in vivo samples. A time-point analysis of the organoids revealed that the cells matured over time as evidenced by a gradual increase in the ChP-specific markers transthyretin and CLIC6 from day 28 to day 40 of culture. Transcriptomic evidence of temporal change in the proportion of mature vs. immature ChP cells confirmed these findings.

These ChP organoids assembled into cyst-like structures containing a colorless fluid that was absent in control organoids. As one of the functions of the ChP in vivo is the secretion of CSF, the researchers compared human CSF samples to the liquid within the organoids by mass spectrometry and found a high degree of similarity between the two. "I would say that the finding that the fluid was so very similar to CSF in vivo was quite surprising. I had expected it to be very different as of course in vivo it is in contact with many other cell types, such as neurons and astrocytes of the brain. But we found a huge overlap between proteins found in CSF in vivo and the fluid generated by choroid plexus organoids, suggesting that the majority of components are indeed coming from the choroid plexus itself," explained Lancaster.

Having established the identity of ChP organoids, the researchers next focused on its ability to function as a barrier. Using transcriptomic analysis and immunofluorescence staining, they confirmed the presence of tight junctions, which are structures formed when two cells adhere and which present a barrier, thus controlling the permeability of the cell layer. The researchers tested the permissivity of the ChP organoids to BIA 10-2474, a compound that previously failed in clinical trials as a result of severe neurotoxicity. Results pinpointed the likely cause of toxicity as an absence of efflux through the $\mathrm{ChP}$ barrier leading to toxic accumulation within the CSF.

Thus, the ability to produce this vital biological fluid in vitro opens up avenues for research into the biology of the bloodCNS barrier and CSF production. The team envisions the use of these organoids not only in testing their permeability to potential new drugs but also as a novel route of drug delivery through the CSF.

"The choroid plexus is heavily understudied, and I think much more research is needed on its function and the CSF more generally. Given how important this biological fluid is for brain function, we know so little. In addition, the bloodbrain barrier gets a lot of focus when it comes to drug permeability or pathogen invasion, but the choroid plexus, or the blood-CSF barrier, is just as important," concluded Lancaster.

Madhura Mukhopadhyay

Published online: 1 September 2020 https://doi.org/10.1038/s41592-020-0950-5

Research paper

Pellegrini, L. et al. Human CNS barrier-forming organoids with cerebrospinal fluid production. Science https://doi.org/10.1126/science.aaz5626 (2020).

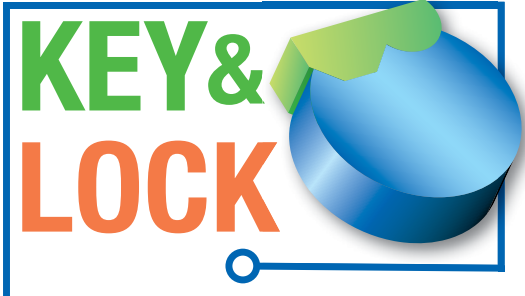 \\ Working Together For Stem Cell Research Innovation}

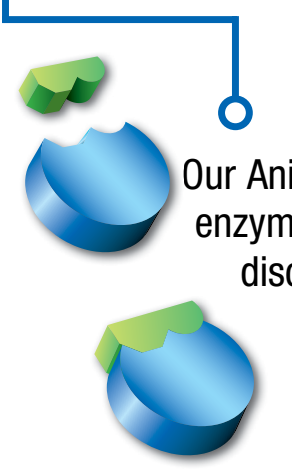

That's why

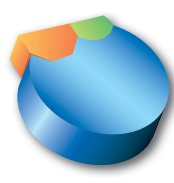

Worthington, the primary enzyme producer, has more citations in respected journals like Nature Methods and rank higher in search engines like $B i o z$.

\section{AF COLLAGENASES 99\% Bioz Rating AF DNASES 92\% Bioz Rating AF STEMxyme ${ }^{\oplus} \quad 95 \%$ Bioz Rating}

\section{Unlock the power of Worthington} with our NEW Stem Cell

Research Products catalog. Go to: Worthington-Biochem.com

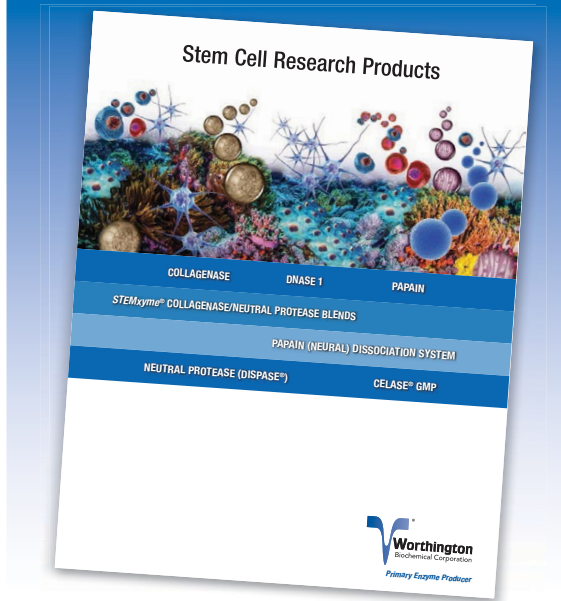

